\title{
http://bjas.journals.ekb.eg \\ Value of Endocan in Diagnosis and Prognosis of Neonatal Sepsis
}

S.A.Dabbour, E.H.Assar and M.A.Abdel-Aleem

Pediatrics Dept., Faculty of Medicine, Benha Univ, Benha, Egypt

E-Mail: midooo20028@yahoo.com

\begin{abstract}
Sepsis is one of the most common causes of morbidity and mortality in the newborn. Early diagnosis and treatment is vital to improve outcome. Investigate the possible role for endocan in the diagnosis of neonatal early onst sepsis. 40 children were included in the study, 20 septic and 20 controls, all patients were subjected to full history and clinical assessment and labouratory tests as CBC, CRP and serum level of endocan by ELISA. Results: Neonatal Sepsis group showed significantly higher Endocan when compared to control group $(\mathrm{p}<0.001)$, ROC curve of Endocan level was conducted for discrimination between neonatal sepsis cases and control groups. Endocan showed AUC (AUC=0.758). At cut off value of 21.4, sensitivity was 70\%, specificity was $78.3 \%$, PPV was $74.5 \%$, NPV was $68.1 \%$, accuracy was $70.8 \%$. Serum endocan levels are significantly increased in septic compared to non-septic neonates in the early stages of sepsis. We suggest that endocan is an effective, reliable, and promising new biomarker for detecting neonatal sepsis.
\end{abstract}

Key words: Endocan, Neonatal sepsis.

\section{Introduction}

Neonatal early-onset sepsis (EOS) is defined as an invasive infection arising in the first 72 hours since birth, but actually approximately $90 \%$ of the cases occur within the first 24 hours of life [1]. The reported incidence of EOS for premature neonates is about $1 \%$ with a mortality rate of up to $30 \%$ [2]. Positive blood cultures are the gold standard for the confirmation of sepsis, but time to results usually exceeds 24 hours and reported sensitivity is low [3].

EOS diagnostic assessment is based on the history of pregnancy and delivery and nonspecific clinical signs such as poor feeding, persistent crying or irritability, tachycardia skin mottling, respiratory distress, apnea, lethargy, hypotension or temperature instability $[1,3]$. This multitude of potentially suggestive signs and symptoms make EOS diagnosis difficult, thus leading to therapeutic delays.

Early initiation of antimicrobial therapy has the potential to decrease the morbidity and mortality [3], but given the inherent subjectivity of clinical elements, there is a continuous search for new objective methods of early diagnosis.

Endocan (endothelial cell-specific molecule1), a proteoglycan expressed by lung and kidney endothelial cells, detectable in the blood stream [4] might be useful as a biomarker for sepsis diagnosis. In vitro experiments showed that proinflammatory cytokines like interleukin (IL)-1 $\beta$ and tumor necrosis factors (TNF)-alpha, upregulate the endocan messenger RNA and subsequently induce its secretion from endothelial cells.

The aim of this study is to investigate a possible role for endocan in the diagnosis of neonatal EOS, thus facilitating timely initiation of treatment and improving the outcome of these critically ill newborns.

\section{Subjects and methods}

This study was carried out on children attending the Neonatal Intensive care Unit (NICU) at Benha Children Hospital during the period from June to November 2017.

The present study inlcuded 40 children; classified into two groups:

- Septic Group:sixty (20) children with clinical sepsis admitted to NICU .

- Control Group:sixty (20) healthy children.

Any neonate admitted to NICU with proven sepsis by laboratory work up. Neonates were evaluated for clinical manifestations and Laboratory markers of neonatal sepsis

- Gestational age; full term and preterm neonates.

- Male and female neonates.

\subsection{All patients were subjected to :}

- Complete history taking and clinical examination including: Vital signs, general appearance, demographic features and anthropometric measurements including (weight, length, and head circumference), gestational age assessment using New Ballard score, APGAR

- Sampling: From each newborn included in the study, $1 \mathrm{~mL}$ of blood was collected from a peripheral vein on the first day of life, at admission in the neonatal intensive care unit (NICU) at the time of initial laboratory workup before any treatment. Serum was immediately isolated and frozen at $-80^{\circ} \mathrm{C}$ until analysis. The concentration of endocan was determined by a sandwich-type enzymelinked immunosorbent assay using antiEndocan monoclonal antibodies (Do It Yourself ELISA Kit H1, Lunginnov, Lille, France). Values are expressed in $\mathrm{ng} / \mathrm{mL}$ [6].

- Routine investigations: as Complete blood count (CBC); Serum C-reactive protein (CRP) 
by tubidmetry.; Renal function tests (urea and creatinine) ;Liver function tests : serum glutamic oxaloacetic transaminase (SGOT) and serum glutamic pyruvic transaminase ( SGPT ).

\subsection{Statistical analysis}

The collected data was revised, coded, tabulated and introduced to a PC using Statistical package for Social Science (IBM Corp. Released 2011. IBM SPSS Statistics for Windows, Version 20.0. Armonk, NY: IBM Corp.). Data were presented and suitable analysis was done according to the type of data obtained for each parameter.

\section{Results}

The present study included 20 patients with neonatal sepsis and 20 healthy control groups.

There was no significant difference between the two groups regarding age and sex, table 1 . and Mothers of neonates with sepsis had significantly higher frequency of PROM when compared to control group. So there was a significant difference between the two groups regarding PROM

Neonatal sepsis group had significantly lower birth weight, current weight, APGAR 1, 5; significantly higher frequency of preterm labor, LBW, lethargy, poor feeding, pallor, jaundice and hypothermia when compared to control group. Mode of delivery did not differ significantly between both groups.

Table (1) Comparison of demographic data between all studied groups.

\begin{tabular}{lllllll}
\hline & & \multicolumn{2}{c}{ Control } & \multicolumn{2}{l}{ Neonatal Sepsis } & p \\
& & $\mathbf{N = 2 0}$ & & $\mathbf{N = 2 0}$ & \\
\hline Age (years) & mean+SD & 16.6 & 5.2 & 15.9 & 4.6 & $0.549^{\mathrm{T}}$ \\
Male & $\mathbf{N}, \boldsymbol{\%}$ & 15 & $75 \%$ & 16 & $80 \%$ & $0.297^{\mathrm{C}}$ \\
Female & $\mathbf{N}, \boldsymbol{\%}$ & 5 & $25 \%$ & 4 & $20 \%$ & \\
\hline
\end{tabular}

Mean NICU stay was 19 days, ranged from 10 to 33 days

Table (2) Comparison of laboratory data among all studied groups.

\begin{tabular}{|c|c|c|c|c|c|c|c|c|}
\hline & & \multicolumn{3}{|c|}{ Control } & \multicolumn{3}{|c|}{ Neonatal Sepsis } & \multirow[b]{2}{*}{$\mathbf{p}$} \\
\hline & & $\mathrm{N}=60$ & & & $\mathrm{~N}=60$ & & & \\
\hline Urea $(\mathrm{mg} / \mathrm{dL})$ & mean \pm SD & 10.3 & \pm & 3.0 & 49.5 & \pm & 16.2 & $<0.001^{\mathrm{T}}$ \\
\hline Creatinine (mg/dL) & mean \pm SD & 0.6 & \pm & 0.1 & 0.8 & \pm & 0.1 & $0.201^{\mathrm{T}}$ \\
\hline $\operatorname{AST}(\mathbf{U} / \mathbf{L})$ & mean \pm SD & 16.1 & \pm & 3.4 & 114.3 & \pm & 33.1 & $<0.001^{\mathrm{T}}$ \\
\hline $\operatorname{ALT}(\mathbf{U} / \mathbf{L})$ & mean \pm SD & 18.7 & \pm & 3.2 & 76.5 & \pm & 24.0 & $<0.001^{\mathrm{T}}$ \\
\hline CRP (mg/L) & mean \pm SD & 3.5 & \pm & 1.1 & 69.5 & \pm & 10.6 & $<0.001^{\mathrm{T}}$ \\
\hline
\end{tabular}

SD, standard deviation; T, Student $t$ test.

. Mean Endocan in control group was $18.1 \mathrm{ng} / \mathrm{mL}$, ranged from 11.2 to 24.7 , while it was $25.3 \mathrm{ng} / \mathrm{mL}$, ranged from 11.3 to $36.3 \mathrm{ng} / \mathrm{mL}$ in neonatal sepsis cases. Neonatal Sepsis group showed significantly higher Endocan when compared to control group ( $\mathrm{p}<0.001)$, Fig (1).

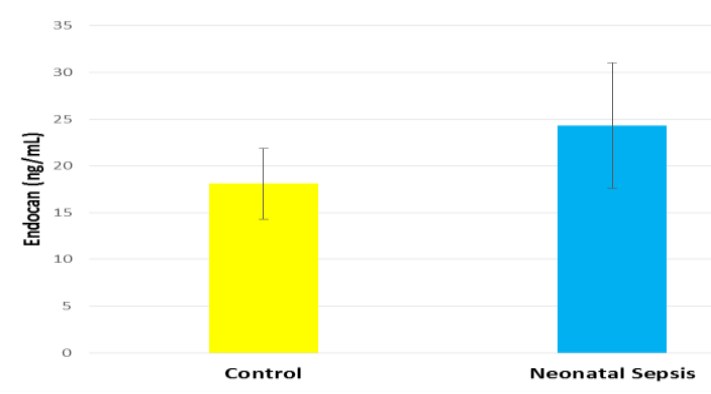

Fig(1) Column chart for Endocan concentration in all studied groups. Bar represents mean, while error bar represents standard deviation. 
Neonaltal sepsisgroup showed significantly higher CRP (69.5) than the control group (3.5). p=0.001, fig(2)

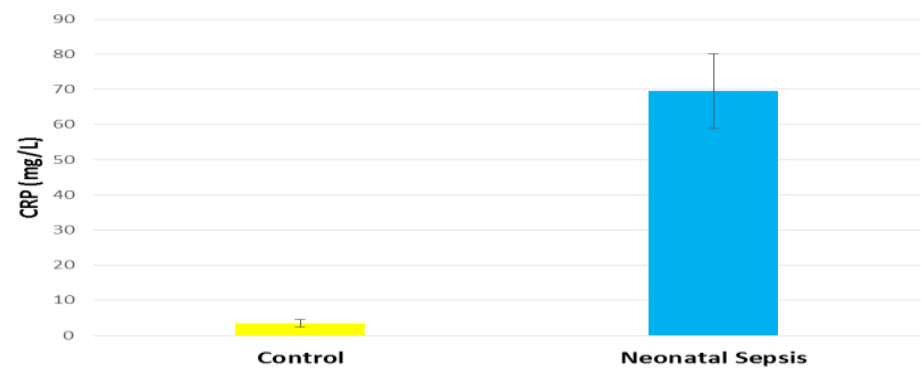

Fig (2) Column chart for CRP in all studied groups.

ROC curve of Endocan level was conducted for discrimination between present and absent bacterial culture growth. Endocan failed to discriminate between present and absent bacterial culture growth .AUC (AUC=0.586). At cut off value of 23.75 , sensitivity was $50.9 \%$, specificity was $71.4 \%$, PPV was $93.1 \%$, NPV was $16.1 \%$, accuracy was $53.3 \%$.

Fig(3)

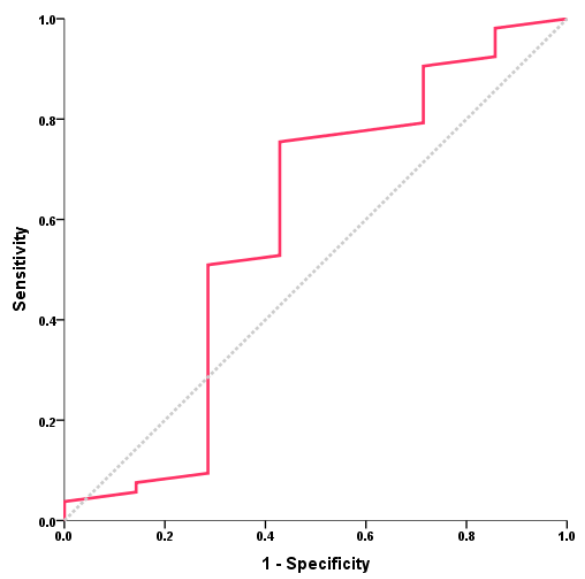

Fig (3) ROC curve of Endocan for discrimination between present and absent bacterial culture growth.

\section{Discussion}

Neonatal sepsis is widely recognized as a clinical syndrome of systemic inflammation in response to, or occurring the same time as, a possible or proven infection (frequently by identifying bacterial bloodstream infection) occurring in children $\leq 28$ days of age. A consensus definition of neonatal sepsis has remained a challenge [7].

In our study, male patients represented $78.3 \%$, while females represented $21.7 \%$. Our study is in agreement with [8], [9] as they reported that there was male sex predominance and the male-female ratio was 2:1 and 1.3:1 respectively. Incidence of septicemia was higher in males than in females. This is possible because the factors regulating the synthesis of immunoglobulin are situated on the Xchromosome [10].

Mothers of neonates with sepsis had significantly higher frequency of PROM when compared to control group. Similar observations have been reported elsewhere by Jabiri and his colleagues, as denoted that early membrane rupture exposes a newborn to ascending microorganisms from the vagina into the amniotic sac and if not managed well, neonate can develop sepsis after delivery [11]. Contrary to our findings, a study by Siakwa and his colleagues did not find premature rupture of membrane as a significant risk factor; probably due to appropriate interventions put in place to manage such cases [12].

In the present study, mode of delivery showed C.S was more prevalent than V.D as C.S represented $56.7 \%$ of the sepsis group. In agreement to our study [12], where they found mode of delivery not to be statistically related with neonatal sepsis Our results were in disagreement with [13] found C.S delivery as a variable that was statistically associated with the risk of developing neonatal sepsis, which is consistent with the results of the current study. 
Regarding the gestational age, our study showed that the mean \pm SD of gestational age was $36.5 \pm 1.7$ weeks and $50 \%$ of cases were less than 38 week GA also, we noted that sepsis group had higher frequency of preterm neonates than the control group. This is in agreement with the finding from an earlier study done by [14]. This could be explained by the fact that premature newborns have poor host defenses and thus are more likely to suffer from neonatal sepsis.

The study demonstrated that low Apgar score (after 1 minutes and after 5 minutes) was significantly associated with neonatal sepsis. One previous study has also reported similar result [15]. Neonates with low Apgar score tend to have poor adaptation to extra uterine life due to the stress experienced during labour and therefore are more prone to infection [6].

Various hematological parameters, a complete blood count, white blood cell count and differential, immature neutrophil count, band form count and platelet count and immature/mature neutrophil count ratios individually and in combination have been evaluated for their ability to predict neonatal sepsis [16].

C-reactive protein is an acute phase protein which may be useful in the early diagnosis of neonatal sepsis as it rises as much as a thousand fold within 4 to 6 hours of an inflammatory process [17].

In this regard we found that CRP was significantly increased compared to control group.

[18] found that CRP identified 134 out of 181 neonates who had culture-proven sepsis, with sensitivity of $74 \%$ and a negative predictive value of $79 \%$, implying that close to three quarters of neonates with suspected sepsis will be correctly diagnosed using CRP. This means that one out of every four neonates with sepsis will be missed. This is much too high to base the decision not to start empirical antibiotics for a neonate with suspected sepsis.

In the present study we found that platelet count was significantly decreased in the sepsis group. In accordance with our findings El-Mashad and his colleagues denoted that although the mean platelet count was above the conventional normal range of 150,000 , platelet count has been significantly less in the sepsis group [19].

In the present study the mean Endocan in control group was $18.1 \mathrm{ng} / \mathrm{mL}$, ranged from 11.2 to 24.7, while it was $24.3 \mathrm{ng} / \mathrm{mL}$, ranged from 11.3 to $36.3 \mathrm{ng} / \mathrm{mL}$ in neonatal sepsis cases. Neonatal Sepsis group showed significantly higher Endocan when compared to control group $(\mathrm{p}<0.001)$.

In accordance with our findings, The significant decrease of serum endocan level was found $t$ be compatible with the resolution of the systemic inflammatory response following specific treatment, as there are data linking the synthesis and release of endocan to pro-inflammatory cytokines [21]. indicating that endocan is useful when monitoring treatment response.

Endocan has also been considered a prognostic factor for sepsis in sepsis in adults because it shows significantly greater increases with sepsis severity and mortality [22].

This can be explained as elevated serum endocan levels in sepsis reflect endothelial activation, which is a significant pathophysiological change associated with inflammation [24]. Endocan inhibits leukocyteendothelial cell adhesion, a critical step in the migration of leucocytes into tissue, thereby reducing tissue damage by impeding leukocyte migration into tissue [21].

\section{Conclusion}

serum endocan levels are significantly increased in septic compared to non-septic neonates in the early stages of sepsis; the levels were significantly associated with mortality and longer hospital stay.

This suggests a potential role for endocan serum level as a marker for early diagnosis and follow-up in neonatal sepsis. So, we suggest that endocan is an effective, reliable, and promising new biomarker for detecting neonatal sepsis.

\section{References}

[1] K. Edmond and A. Zaidi, "New approaches to preventing, diagnosing, and treating neonatal sepsis," PLoS Med., Vol. 7, PP. e1000213, 2010.

[2] M. E. Abdelhameed, "Assessment of cell-free fetal DNA level in maternal plasma in cases of spontaneous preterm labor," CU Theses, vol 13,PP.78-80,2012.

[3] G. Du Pont-Thibodeau, J.-S. Joyal, and J. Lacroix, "Management of neonatal sepsis in term newborns," F1000Prime Rep., Vol. 6, PP.88-90,2014.

[4] A. Camacho-Gonzalez, P. W. Spearman, and B. J. Stoll, "Neonatal infectious diseases: evaluation of neonatal sepsis," Pediatr. Clin. North Am., Vol. 60, PP. 367, 2013.

[5] G. I. Zonda, R. Zonda, A. T. Cernomaz, L. Paduraru, A. L. Avasiloaiei, and B. D. Grigoriu, "Endocan-a potential diagnostic marker for early onset sepsis in neonates," J. Infect. Dev. Ctries., Vol. 13, PP. 311-317, 2019.

[6] K. A. Simonsen, A. L. Anderson-Berry, S. F. Delair, and H. D. Davies, "Early-onset neonatal sepsis," Clin. Microbiol. Rev., Vol. 27, PP. 21-47, 2014.

[7] N. D. Toan , "Clinical features, antimicrobial susceptibility patterns and genomics of bacteria causing neonatal sepsis in a children's hospital in Vietnam: protocol for a prospective observational study," BMJ Open, Vol. 8, 
PP.e019611, 2018

[8] F. A. S. MORTAZAVI, S. S. Hosseinpour, and N. Nejati, "Acute kidney failure in neonatal period," 2009.

[9] D. Youssef, H. Abd-Elrahman, M. M. Shehab, and M. Abd-Elrheem, "Incidence of acute kidney injury in the neonatal intensive care unit," Saudi J. kidney Dis. Transplant., Vol. 26, PP.67, 2015.

[10] K. A. Kuruvilla, S. Pillai, M. Jesudason, and A. K. Jana, "Bacterial profile of sepsis in a neonatal unit in south India," Indian Pediatr, Vol. 35, PP. 851-858, 1998.

[11] A. Jabiri, H. L. Wella, A. Semiono, A. Saria, and J. Protas, "Prevalence and factors associated with neonatal sepsis among neonates in Temeke and Mwananyamala Hospitals in Dar es Salaam, Tanzania," Tanzan. J. Health Res., Vol.18,PP.90-92, 2016.

[12] M. Siakwa, D. Kpikpitse, S. C. Mupepi, and M. Semuatu, "Neonatal sepsis in rural Ghana: A case control study of risk factors in a birth cohort,Vol.31,PP.98-99,2014.

[13] M. T. Utomo, "Risk factors of neonatal sepsis: a preliminary study in Dr. Soetomo hospital," Indones. J. Trop. Infect. Dis., Vol. 1, PP. 2326, 2010.

[14] K. N. Haque, "Necrotizing enterocolitis-Some things old and some things new: A comprehensive review," J. Clin. Neonatol., Vol. 5, PP. 79, 2016.

[15] V. Sundaram et al., "Blood culture confirmed bacterial sepsis in neonates in a North Indian tertiary care center: changes over the last decade," Jpn J Infect Dis, Vol. 62, no. 1, PP. 46-50, 2009.

[16] V. Manucha, U. Rusia, M. Sikka, M. M. A. Faridi, and N. Madan, "Utility of haematological parameters and C-reactive protein in the detection of neonatal sepsis," J. Paediatr. Child Health, Vol.38, PP.459-464, 2002.

[17] J.-H. Weitkamp and J. L. Aschner, "Diagnostic use of C-reactive protein (CRP) in assessment of neonatal sepsis," Neoreviews, Vol. 6, PP. e508-e515, 2005.

[18]B. A. West, O. Peterside, R. O. Ugwu, and A. U. Eneh, "Prospective evaluation of the usefulness of C-reactive protein in the diagnosis of neonatal sepsis in a sub-Saharan African region," Antimicrob. Resist. Infect. Control, Vol.1, PP.22, 2012.

[19] G. M. El-Mashad, H. M. El-Sayed, and O. H. Salem, "Serum leptin level as a marker of neonatal sepsis," Menoufia Med. J., Vol. 29, p. 252, 2016.

[20]M. Saldir,"Endocan and soluble triggering receptor expressed on myeloid cells-1 as novel markers for neonatal sepsis," Pediatr. Neonatol., Vol. 56, PP. 415-421, 2015.

[21]D. Béchard , "Human endothelial-cell specific molecule-1 binds directly to the integrin CD11a/CD18 (LFA-1) and blocks binding to intercellular adhesion molecule-1," J. Immunol., Vol. 167, PP. 3099-3106, 2001.

[22]D. Pauly, "Endothelial cell-specific molecule1/endocan: diagnostic and prognostic value in patients suffering from severe sepsis and septic shock," J. Crit. Care, Vol. 31, PP. 68-75, 2016.

[23]A. Scherpereel et al., "Endocan, a new endothelial marker in human sepsis," Crit. Care Med., Vol. 34, PP. 532-537, 2006.

[24] A. Kali and K. S. R. Shetty, "Endocan: a novel circulating proteoglycan," Indian J. Pharmacol., Vol. 46, PP. 579, 2014.

[25] M. Buyuktiryaki et al., "Can Endocan Predict Late-Onset Neonatal Sepsis?," J. Pediatr. Infect. Dis., Vol. 14, PP. 96-102, 2019. 